much complained of when she was examined. Apart from increase in suffering there was no difference in her condition otherwise. The temperature and pulse rate remained steady.-8th: To-day her aspect has changed; her face wears an anxious expression; her eyes are sunk, and she is evidently worse. Has had a bad night, with return of vomiting. The skin feels a little cold. The temperature in the mouth is $99^{\circ}$; her pulse is 140 , feeble, and indistinct in character. The outline of the tumour is not nearly so plain, and there is considerable increase in the area of abdoninal tenderness. The accident which had happened to her was very evident, and operation was spoken of, but was, unhappily, not entertained. Stimulants were administered, the opium pushed, and such means employed as are much useful in rallying from depression. In the evening she was in the same state. There had, however, been no return of the vomiting, and the abdominal tenderness had not undergone notable increase. - 9th : This morning she is somewhat better; her temperature is still $99^{\circ}$, but her pulse has fallen in number, and is of fuller character. The abdominal condition was unchanged; but as it was now evident to her relatives that her best, and indeed only, chance of recovery lay in a surgical operation, they consented to its performance. Through a median abdominal incision there were not observed the evidences of a general peritonitis, but when the small intestine was gently pulled aside to admit of the introduction of the fingers, it was found to be intensely injected and coated at points with thick lymph. When the coils adherent to the abdominal wall were separated in order to reach the crecum, a drachm or two of bad-smelling pus passed along the detaching fingers, and with it an orange pip and a raisin pip. An attempt to bring the cæcum forward failed on account of firm adhesions, and I therefore cut down upon it by a slightly curved incision external to the line of the deep epigastric artery. Then it was readily turned out, and in the middle of the appendix was found an ulcerated opening with greyish edges, through which the pips had passed. The proximal and distal ends were now examined through this opening, but without discovering the presence of concretions or other obstruction; the sloughing edges were cut away, and as the appendix was up to its extremity very closely adherent to the colon it was not removed, but its divided ends were thoroughly occluded by Lembert's stitch. The abdominal cavity was washed out with warm boracic lotion and the incision closed. She rallied well from the shock, and was in the evening easy and comfortable; but in the course of the night the vomiting returned, and became of that gulping character of such evil omen in cases of peritonitis, and she sank on the following day. Only after the removal of the pips were we told that at Christmas she had, after having eatem freely of raisins, had an attack of pain similar to that with which her fatal illness was ushered in, and that between that time and March 6th she had made occasional complaints of cramp in the right iliac fossa.

I have selected these three cases from a very considerable number that have fallen under my observation within the last few years, and I have done this because they will serve as examples of the various ways in which creal and peri-cæeal harm may be provoked.

(To be concluded.)

\section{"IT IS TO THE LYMPHATIC SYSTEM AND CELL AGENCY THAT MOST, IF NOT ALL, FORMS OF DISEASE ARE DUE."}

By W. GROOM, B.A., M.D. CaNTAB., M.R.C.S.E., L.S.A.

IN every variety of disease to which the human body is liable we have a direct cause producing a definite result, and to determine the exact nature of the disease both the cause and its result have to be taken together into consideration. Thus fever is a symptom or result of some agency in the body producing that condition; but to constitute scarlet fever we must have these symptoms or results take a more or less definite course, be of a definite character, and dependent upon a specific agency. Hence in scarlet fever, and also every other variety of disease, we have an agency or exciting cause and results or symptoms: originating therefrom. Between these a definite period of variable duration exists, known as the latent period, and it is during this period that I believe highly important changes take place. For example, in the ordinary operation of vaccination a definite material is introduced into the body at a certain spot, and no immediate results are visible, and it is only after the lapse of a certain period of apparent quiescence that a definite local result manifests itself, and this gradually takes a progressive course, accompanied with a distinct constitutional effect.

The question arises, What is it that occurs between the inoculation and the commencement of the papular formation with its attending febrile symptoms? To arrive at a possible answer to this question, we must first bear in mind the nature of the lymph inoculated. This is a slightly viscid, clear, and transparent fluid, with alkaline reaction and little or no smell, and when viewed with the microscope is seen to have a clear liquid portion or plasma, and a solid portion made up of corpuscular elements which float in the plasma. or lymph; these are few in number, of somewhat rounded but irregular outline, and correspond in all particulars to a description of the corpuscles found in the lymph of the lymphatic system, and both of these are not far removed in character from that of an embryonic protoplasmic cell. In the next place, we must recollect that the seat of inoculation is constructed of cells, arranged with varying regularity in layers; the lowermost of these, belonging to the epidermis, are elongated in shape and perpendicularly disposed upon the dermis, and with their extremities intimately connected with the corresponding irregularities of the dermis. Immediately above these, the cells are of more rounded shape and are furrowed, and so arranged that these furrows approximated together form little channels. Above these we have the Hattened cells which form the upper and denser portion of the epidermis. With the exception, therefore, of these latter layers, the epidermal cells are sufficiently loosely packed together as to leave interspaces, however small, between them; and, moreover, in these spaces leucocytes or corpuscles similar in structure to those spoken of in vaccine lymph and the lymphatic system may here and there be observed, and they also contain a fluid plasma. The dermis or subcutaneous tissue also shows, 'on close examination, the existence of similar spaces, with their cells and plasma, and continuous above with those of the epidermis, and below in the closest contact, if not continuous, with the lymphatics. These spaces may therefore be looked upon as the very commencement of the lymphatic system. Now, in vaccination, these spaces receive some at least of the inoculated vaccine lymph ; for, if the lancet wounds the bloodvessels in its course, it has first passed through spaces existing above them, and, as the blood current is rapid and therefore does not afford sufficient repose for developmental changes to take place in it, we must, I think, conclude that such changes as do take place occur in these lymph spaces. In their ordinary course of life the lymph cells grow and multiply, and in their growth assimilate materials from and modify the character of the plasma in which they live, in much the same way as a torula cell of yeast assimilates material from the saccharine. solution in which it grows during the process of fermentation. and converts that solution into alcohol. When, therefore, the plasma derived from a vaccine vesicle is deposited in a lymph space, it mixes with the plasma already existing there, and the cells in these spaces now live in material much of which is the product of vaccine lymph cells. In their growth and physiological functions they assimilate and build themselves up with this material, and so get impressed upon them the same characters as the cells of vaccine lymph-as Dr. Creighton has called it, become spermatised. These cells then, in their turn, modify the plasma of the next space (remembering that the spaces are virtually continuous), and so on, until, by an onward progress from the periphery inwards, varying in its extent and speed according. to the virulence or specitic strength of the inoculated cells or cell products, the whole lymphatic system becomes. spermatised and brought into a similar condition to the foreign agency introduced. We need now only recollect the intimate connexion between the lymphatic system and the vascular system, to understand how the whole blood-vascular system generally becomes, in the more virulent varieties, infected. Since the vaccine lymph inoculated is foreign to its new situation, it acts as an irritative agent, producing a local and general inflammatory result, but tainted with the 
peculiarities of the disease from which it is derived. Looking further into the matter, let me again state that the vaccine lymph vltimately infects the whole system as above described, and so long as this general infection remains in the system, any subsequent inoculation with vaccine lymph is unable to bring about the same definite result, since it is no longer foreign to the plasma of the spaces then receiving it ; but so soon as this influence has died away or been worked out, any subsequent vaccine lymph inoculated would have the same power again, varying in extent, however, with the greater or lesser loss of the influences. In vaccination the accompanying symptoms are weak in intensity on account of the weak spermatising influence of the vaccine lymph. They are febrile in character, and are no doubt due to an altered condition of the blood, brought about by the changres in the lymphatic system being conveyed by the Iymphatics into the bloodvessels. As the contagium of variola can only produce variola of a like kind, so also the contagium of a definite exanthematous affection can only produce the skin eruption peculiar to its progenitor. It would seem that the specific fevers vary somewhat in the influencing power of their contagia ; in many it seems to be life-long, and hence it is that one attack of these gives immunity from subsequent ones. But we must recollect that there is always a tendency for this influence to diminish by age, and that therefore in some cases it sufficiently disappears to render the subject liable to a further invasion of this particular disease. When from ill health the physiological activity of the lymphatic cells in the system is diminished in power, it is naturally even easier for a contagium to attack them than when in perfect health. Hence it is that women after parturition so readily contract scarlet fever. Also when so reduced in strength from nerve influence or other causes their products suffer and are weak, if not abnormal in constituents, and these may therefore develop diseases without any external agency whatever; hence the connexion between parturition and phlegmasia dolens.

Taking the above-stated view respecting the lymph spaces and their connexion with the lymphatic system, we are enabled to state that this system has an extremely wide distribution throughout the human body; existing, in fact, not only in the cutaneous and subcutaneous tissues, but also internally it is found in the follicles of the lymphatics, Malpighian corpuscles of the spleen, Peyer's patches and solitary glands of the intestine, follicles of the pharynx tonsils, trachoma, glands of the conjunctiva, also around bloodvessels, in the pia mater, smaller bronchi, beneath the pleural endothelium and also that of the peritoneum alimentary mucous lining, and medulla of bones. From this immensely wide distribution, therefore, we have no difficulty in understanding how easily the Iymphatic system can be reached from without, and that the contagium of a disease need not necessarily be artificially inoculated to gain an entrance into it. Scarlet fever, for instance, seems to gain entrance by the throat and respiratory tract. In measles the conjunctivæ seem to have a very early primary connexion with the specific contagium. In typhoid fever it would seem to gain adnission by the intestinal tract, judging from the lesions of the agminated and solitary glands and secondary involvement of the mesenteric glands. Passing from the so-called specific fevers, we may next mention syphilis; and here we also have a distinct inoculation in the neighbourhood of the lymphatic system, and the neighbouring lymphatic glands are soon involved; and further, before the characteristic eruptions malie their appearance, there is a distinct latent period in which changes such as I have described can go on ; moreover, we know also the beneficial effect of mercurial inunction on this disease. In syphilis, however, the specific influence seem extremely tardy in working itself out. Again, in pyæmia we find the seat of primary mis chief to be some local abrasion or wound accidentally or surgically made, or after parturition, and in all of these the connective tissues and lymphatics are early involved; and although cases do occur in which no such lesion seems apparent, we may still suppose that the virus can reach the lymphatics by the respiratory tract. In elephantiasis græcorum, the cellular matter which infiltrates the affected tissues is probably developed from the connective tissue cells and leucocytes. In ague, the spleen is soon and sometimes permanently involved, and it will be remembered that this organ is intimately connected with the lymphatic system. In skin affections we can also show forth this lymphatic connexion. 'Thus in erysipelas the tonsils are often the seat of prenonitory inflammation; the erysipelatons swelling contains lymph and corpuscles, the neighbouring lymphatics are enlarged and tender, and the blood contains a distinct increase in the number of its white corpuseles. It would appear, therefore, that in those forms of disease, at least, which are recognised as the result of a contagium, the lymphatic system seems to be tlie chief seat of the more important changes which go on during the so-called latent period, and that the definite symptoms which follow are results of these changes conveyed by the medium of the bloodvessels to the several organs and other parts of the body. Taking now into consideration other varieties of disease, such as tubercle and tumours, we still find the lymphatic system connected with their derelopment or spread, for there seems to be but little doubt that pulmonary tubercle has its origin in the inter-alveolar septa and parietes of the bronchioles, in which situations are found embryonic cells and leucocytes in large numbers; and, further, the spread of tubercle follows a lymphatic tract, as in those cases in which a caseous lymphatic gland is the source of generalised tuberculosis; also we know that the mucous membrane of the intestinal tract, a part most closely connected with the lymphatics, is a common seat of tubercle.

As I have stated, it is from the product of a cell's activity, in its turn affecting or spermatising other cells in its immediate contact by their assimilation of this abnormal product (ordinary lymph being the normal medium of a healthy leucocyte), that all the subsequent changes are probably due; it need not of necessity in every instance be the product itself that gains admittance into the body to act as the germ of a disease, but the particular cell manufacturer itself may in some instances enter and exert its direct influence, therein, or even the normal leucocytes or cell elements of the body may, by abnormal irritation or nerve influence, have their physiological characters changed, and the lymph therefore in which they grow will by their assimilative and productive process be likewise ultimately changed in a corresponding manner. It is by this latter method that $I$ would explain the enlargements of lymphatics from distal irritation, and also the possibility of developing tubercle artificially by other material than the products of tuberculosis, as Sanderson and others have long ago shown. Also this will, I think, in some manner explain the connexion between a sudden shock and subsequent development of disease dependent thereon, such as we now see from railway accidents, and such as I believe to have been the case in a young patient of mine who died of localised meningitis, which gradually developed itself in a previously perfectiy healthy person, with no trace whatever of tubercular history, soon after receiving a serere shock by witnessing the accidental death of a young friend whom he was chasing in the dark, and who, forcibly running against a water hydrant on the roadside, received such internal injuries as to cause rapid death. Again, there may be in some cases an hereditary tendency for leucocytes or cell elements to take on an abnormal growth at a fitting opportunity afforded by ill health or the decadence of life, implanted upon them by the parent just in the same way as features and peculiarities are implanted on the offspring of man and animals. Also the foregoing ideas do not exclude bacteria as a source of disease, they being equally living cells and bringing forth their own peculiar products. Finally, we know that tumours have a cellular origin, and in one class at least-viz., the carcinomata-the lymphatics are most intimately connected with their growth and spread, for the alveoli of cancers may be regarded as the dilated origins of the lymphatic system. Whether these can be derived from contagion seems as yet difficult to positively determine, but from cases which have come under my own observation I am personally inclined to beliere it possible. Taking, then, into consideration the above ideas, I desire to maintain that it is to the lymphatic system and cell agency that most, if not all, forms of disease are due. Wisbech.

NEW INFIRMARY, LANCASTER-A long-needed want is about to be provided for Lancaster by the erection of a new infirmary. Mr. Williamson, M.P., has generously offered $£ 5000$ towards the building fund, and the committee, being thus encouraged, have determined to purchase a site eligibly situated in close proximity to the town, which forms a part of Springfield Park. To erect a suitable building and provide a permanent sustentation fund, it is expected that between $£ 10,000$ and $\mathfrak{f 1 5}, 000$ will be required. 